\title{
Adapting Double Weaving and Yarn Plying Techniques for Smart Textiles Applications
}

\author{
Laura Devendorf \& Chad Di Lauro \\ ATLAS Institute \& Information Science, Electrical and Computer Engineering \\ University of Colorado, Boulder \\ \{laura.devendorf, chad.dilauro\}@colorado.edu
}
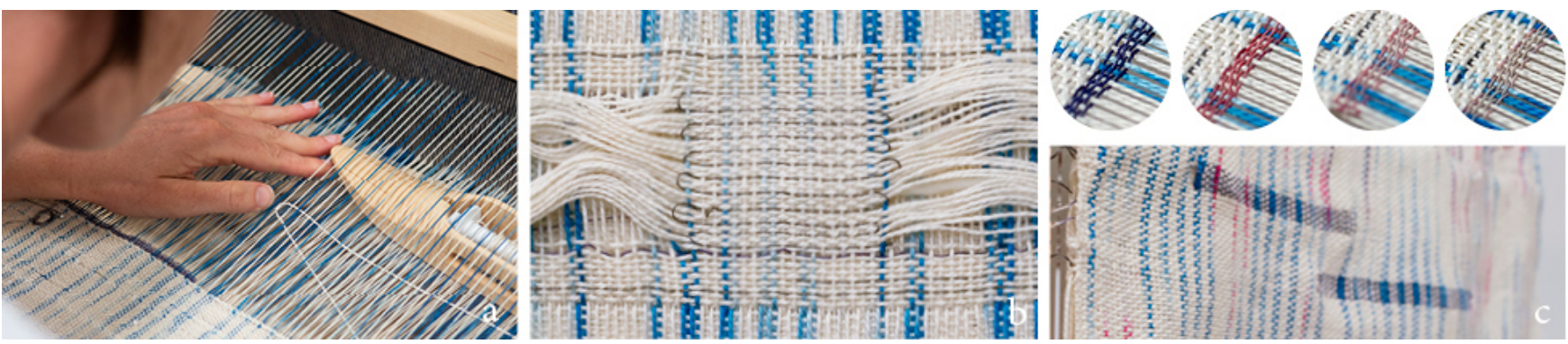

Figure 1: We describe how fiber arts techniques can be adapted for smart textiles applications. (a) We used hand-weaving to create an interactive fabric with thirteen regions that change color in response to touch (b) We used a technique called double weaving to create press buttons that hide the sensing yarns from view. (c) We experimented with plying custom color-changing yarns to achieve optimal color visibility and power efficiency.

\begin{abstract}
Smart textiles integrate sensing and actuation components into their structures to bring interactivity to fabrics. We describe how we adapted two existing fiber arts techniques, double weaving and yarn plying, for the purpose of creating a woven textile that changes color in response to touch. We draw from this experience to make three core contributions: descriptions of our experiments plying yarns that change between three color states; descriptions of double weaving structures that allow us to support interactivity while hiding circuitry from view; and suggestions for how these techniques could be adapted and extended by other researchers to make richly crafted and technologically sophisticated fabrics.
\end{abstract}

\section{Author Keywords}

Smart textiles; Hand-weaving; Yarn plying; Craft;

\section{CCS Concepts}

Human-centered computing $\sim$ Human computer interaction (HCI); Human-centered computing Interface design prototyping

\section{INTRODUCTION}

Smart textiles offer new forms of interactivity by imbuing fabrics with circuitry. We distinguish smart textiles from a broader category of e-textiles by the way smart textile

Permission to make digital or hard copies of all or part of this work for personal or classroom use is granted without fee provided that copies are not made or distributed for profit or commercial advantage and that copies bear this notice and the full citation on the first page. Copyrights for components of this work owned by others than the author(s) must be honored. Abstracting with credit is permitted. To copy otherwise, or republish, to post on servers or to redistribute to lists, requires prior specific permission and/or a fee. Request permissions from Permissions@acm.org. TEI '19, March 17-20, 2019, Tempe, AZ, USA

(C) 2019 Copyright is held by the owner/author(s). Publication rights licensed to ACM. ACM 978-1-4503-6196-5/19/03 ...\$15.00 https://doi.org/10.1145/3294109.3295625 components are realized within the structure of fabrics, at the level of individual yarns. For example, a smart textile force sensor leverages the inherent three-dimensionality of a waffle weave to make a fabric that creates measureable changes in resistance when pressed [18]. We see this as distinct, in terms of production, from approaches that layer premade fabrics to form new kinds of components.

Designers of smart textiles have an increased amount of control over the specific functionality and aesthetic of the textiles they produce. At the same time, weaving smart textiles sets up a unique set of design constraints that are different from more common weaving practices. Specifically, one needs to ensure that particular yarns maintain connectivity (or not) and the yarns with which one weaves may need to be crafted specifically to provide particular properties to the circuit (e.g. a specific resistance or non-standard color changing functionality).

We address these challenges by describing how we adapted double weaving (a technique for weaving multiple layers into a single fabric) and yarn plying (a method for twisting multiple yarns into a single yarn) for the purposes of creating smart textiles. We applied these techniques to create an interactive textile with thirteen distinct regions that correlate touch sensing with color change in the pattern. The touch sensing buttons are distributed throughout the bottom layer of a two-layered fabric, making their circuitry invisible when displayed on a wall (Figure 1b). Pressing those regions changes the color of one of the long thin rectangles in the pattern. A single touch turns a dark purple region to a rich pink, a second touch turns the pink region to white, and a third touch resets the region back to its original purple (Figure 1c). 
Through the experimentation and production of our touch textile, we began to see how the techniques for double weaving and yarn plying could be useful beyond our application. In an effort to bring attention to these techniques and aid the practices of other smart textiles practitioners, we contribute (1) descriptions and findings from our experiments plying custom thermochromic yarns; (2) descriptions and examples of double weaving techniques and (3) visions for future work that could leverage these techniques.

\section{WEAVING SMART TEXTILES}

While embroidery (e.g. [15]), knitting (e.g. [19]), crochet (e.g. [5]), and silk-screening (e.g. [9]) can all be used to bring interactivity to textiles, we focus specifically on weaving: a process by which yarns are interlaced in perpendicular directions to create fabrics. Where embroidery and silk-screening offer design flexibility in terms of patterning existing fabrics and knitting is useful for creating 3-dimenstional or stretchy forms, weaving has some specific advantages for smart textiles applications [3]. Specifically, because woven fabrics tend to be less stretchy than knitted fabrics the processes of weaving places less tension or strain on yarns that are integrated within the fabric. Weaving also makes it easy to integrate yarns that have little to no stretch, such as stainless steel yarn or thin wires. One typically encounters woven fabrics in the form of tailored garments like suits, denim, and/or upholstery.

Artists/designers/technologies have been creating woven smart textiles over the past few decades. Perhaps the earliest example of smart textiles weaving, as we conceptualize it here, occurred in the 1960's when women hand-wove the rope core memory units during the Apollo missions [16]. More recently, in 1997, Rehmi Post and Maggie Orth described smart fabrics emerging from existing practices of weaving with metallic fibers and demonstrate several versions of woven circuits [14]. In 2004, Joanna Berzowska described animating textiles using thermochromic pigments with multiple temperature thresholds, integrated heating wires, and envisioned creating woven circuit boards using double and triple weaving techniques like we describe here [2]. Between 2005 and 2010, Linda Worbin developed a methodology of smart textile design with specific focus on thermochromic display possibilities [17] and collaborated with designer Margot Jacobs to envision new interactions with living "smart" fabrics; Maggie Orth wove a series of thermochromic tapestries that explored the design space of thermochromics as well as double woven structures [13]; Since these foundational demonstrations of smart fabric sensors and displays, several others have extended designers' imagination of how smart fabrics could shape our perception of digital materials [10,20,21] and social relationships $[7,9,12]$. This history laid the foundation for projects like Google's Project Jacquard [8] and Ebb [5], which leveraged weaving to recreate core user interface elements and study how people envisioned engaging with them in daily life.
We complement this history with a specific attention to the techniques employed to create many of these prototypes, which have been used in the past but have not yet been explicitly described in terms intended to spark the imagination of a broader interaction design community. For example, weavers have long created their own yarns with custom properties, used double weaving to pattern textile surfaces, and leveraged these properties for interactive textile displays (e.g. [5,7,22]). However, the focus on such work has emphasized the visual/interactive nature of the design object rather than a detailed description of the specific techniques employed to support those functions. We believe with a better understanding of the fundamental techniques, a broader community will be able to envision and participate in the creation of interactive textile prototypes.

\section{Understanding Weaving, Looms, and Patterns}

Woven fabrics are created on looms, which stretch multiple lengths of yarn (collectively referred to as the "warp") across two beams (Figure 2a). A weaver then works continuous lengths of yarn into a fabric by threading it over and under the warp yarns in a perpendicular direction. The yarns worked into the warp are collectively referred to as the "weft." The particular way the weft travels over and under the warp yarns determines the pattern and structure of the fabric. These patterns are represented by weave "drafts," two-dimensional grids that represent each crossing of a weft and warp thread as a cell within a grid (Figure 2, left). These primitives can be combined with different colors of yarn and in different sequences to create a wide array of patterns and structural possibilities. Some looms, such as the 8-shaft loom we use in this paper, assist in the quick weaving of patterns by allowing one to thread specific sets of warps yarns through different frames or harnesses. These frames are attached, in user specified combinations, to foot pedals called treadles. While weaving one pushes foot pedals in their desired combination, passing the weft through the space created between the raised and lowered yarns (called the "shed"). We found the following books especially helpful in understanding how to weave $[1,4,6]$

\section{WEAVING OUR TOUCH FABRIC}

We developed the techniques we describe in this paper during the process of creating a fabric that would change color in particular regions where the fabric was pressed. The prototype we developed integrates multiple sensing and actuation elements (twenty-six to be exact) into a woven textile. We used 5/2 Pearl Cotton ${ }^{1}$, plyed stainless steel ${ }^{2}$, and cotton covered copper ${ }^{3}$ painted with thermochromic pigments to create this design and wove them on an 8-shaft Schacht Baby Wolf loom warped at 20 warps per inch.

\footnotetext{
${ }^{1} \mathrm{https}: / /$ halcyonyarn.com/yarn/083/52-pearl-cotton-yarn

${ }^{2}$ https://www.kr4.us/Conductive-Thread-60g-Stainless-Steel.html

$3.15 \mathrm{~mm}$ Double Cotton Covered Copper,

sourced from https://wires.co.uk/acatalog/dcc_wire.html
} 


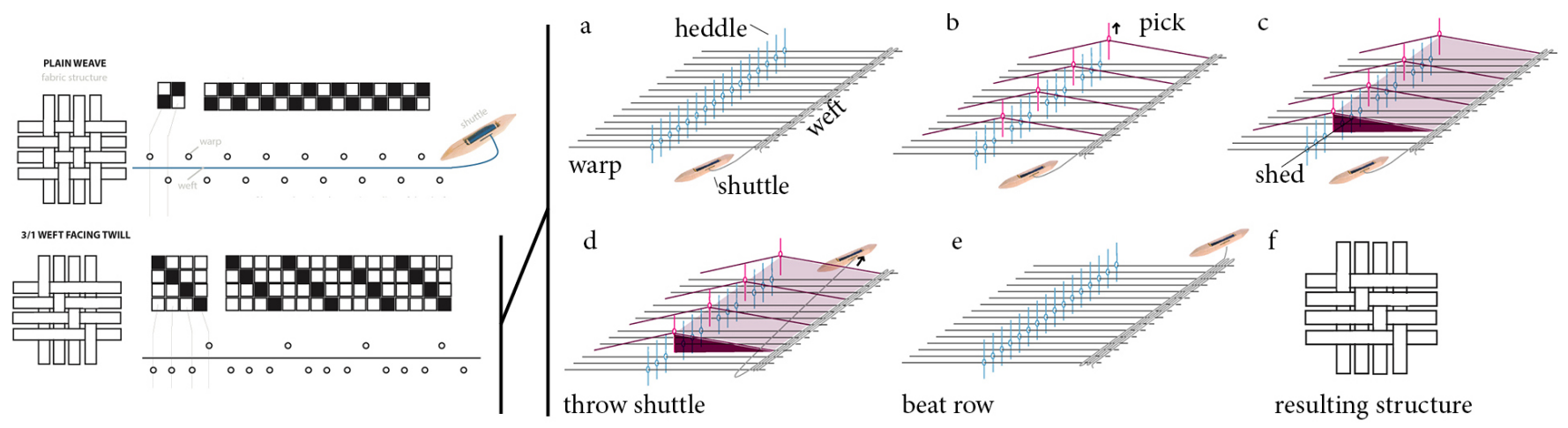

Figure 2: Left: A weaving draft describes which yarns must be raised and lowered to achieve a particular pattern. A cell that is black means the weft travels under the warp. A cell that is white means that the weft travels over the warp. (right) the steps a weaver takes to implement a draft on the loom. Steps b-e repeat for each row of the draft, resulting in a structure like that shown in (f).

We began by weaving test swatches to figure out how to create functional press pads to integrate in the fabric. Simultaneously, we tested a variety of combinations of heating wires and thermochromic painted yarns in order to find yarn compositions that were power efficient with visually noticeable changes between color states. After discovering textile geometries and yarn constructions that were desirable for sensing and color change, we designed a pattern with several of these components to be created on our loom. We quickly learned how difficult it is to envision what a weave would look like prior to weaving samples and started "improvising" designs within the harness and treadling patterns we had already defined to come up with new patterns that satisfied our functional and aesthetic requirements. Through these improvisations, we arrived at designs that we found to be functionally robust and aesthetically appealing. Our result is a fabric that allows someone to press the fabric to change the color in the associated region. We envision the fabric as first step towards an ambient wearable system that records when and where pressures are exerted on the wearer's body, such as the experience of holding children. Detailed patterns and descriptions of how we created the fabric are also available on our project webpage ${ }^{4}$.

In the paper that follows, we will describe our experiments and process of realizing this textile, focusing specifically on our tests with yarn plying and double weaving. In the discussion, we present other promising techniques and speculate on possible directions for future research involving these techniques.

\section{PREPARING THE YARNS}

While prior work has demonstrated the display capabilities of woven fabrics by coating yarns or fabrics with thermochromic pigments, techniques for coating yarns or fabrics and providing heat vary. Our process began with experiments to determine which yarns or combinations of yarns would offer the best color change results and power efficiencies. We focused specifically on studying yarns that coupled resistive heating wires with fibers painted with

${ }^{4} \mathrm{http}: / /$ unstable.design/force-fabric/ thermochromic pigments. We needed a combination that was power efficient, easy (enough) to produce, able to integrate into weaving without breaking, and made the color change very visible. To create this, we experimented with various mixtures of thermochromic paints and yarn constructions:

\section{Yarn Painting}

Because thermochromic color changes do not emit light, and their change in color is gradual, the eye can have difficulty registering the color change as it is taking place. This requires designers to carefully consider the interactions between various colors in the fabric pattern as well as within the transition.

We were able to procure blue and red thermochromic pigments that become transparent at $28^{\circ} \mathrm{C}, 43^{\circ} \mathrm{C}, 56^{\circ} \mathrm{C}$ from Chromatic Technologies Incorporated ${ }^{5}$. Thus, mixing the pigments of varying activation temperatures had the effect of revealing distinct color states as the textile become increasingly warm or cool. While we were able to transition between three unique color states using all temperature pigments, we found that heating yarns to $56^{\circ} \mathrm{C}$ was too resource intensive and uncomfortable for wearable applications. We also discovered that some transitions between colors were more or less noticeable than others. Specifically, a shift from purple to red to white is more visually stunning than a shift from purple to blue to white (Figure 3).

We created our final paint mixture by mixing:

- 3 parts blue thermochromic that activates at $28^{\circ} \mathrm{C}$;

- 1 part red thermochromic that activates at $43^{\circ} \mathrm{C}$;

- 4 parts Liquitex clear acrylic gel medium.
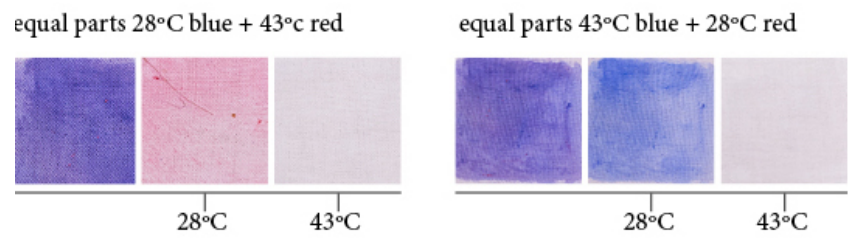

Figure 3: Two different transitions from purple to white vary based on which pigments "disappears" first. 
This creates a paint that looks like a deep blue/purple when it is at room temperature, transitions to a bright pink and then to white (Figure 1). We added three times more blue than red in order to emphasize the color transition from a very deep blue-purple to pink. When heated to $28^{\circ} \mathrm{C}$, the blue pigments become transparent revealing a rich pink. When heated to $43^{\circ} \mathrm{C}$, the red pigments become transparent and reveal the color of the yarn (white in our case).

Painting the yarns is a less than ideal method of applying thermochromic pigments. While we had some success using a solution of vinegar and thermochromic pigments to set the color on wool, the color was dull, did not hold and came off during washing or working in our hands.

\section{Using Plying to Create Heating Wire}

While many thermochromic display projects use heating pads to activate color change (e.g. [9]), there are functional and aesthetic benefits to coupling colored yarns with heating wires. We used a technique called plying, in which two yarns are spun together, to create several combinations of heating wires and fibers. While one can ply yarns by tying two lengths of yarn to a screwdriver, we used a drop spindle to ply our yarns. Plying with a drop spindle has the advantage of allowing one to spin longer continuous lengths of yarn.

We produced custom plied yarns by tying one length of heating wire and one length of wool or cotton yarn to the hook at the end of the spindle then spinning it together. To maintain structural stability and balance, we then took the same yarn we spun, looped the center of it around the spindle hook, and spun it again in the other direction (Figure 4). This made the yarn less prone to unraveling and also integrated more lengths of heating wire per unit length.

We created several samples in which a traditional yarn was plied with a resistive heating wire. We provide each material a unique code to which we will refer in the rest of the section and associated diagram.

The traditional yarns we tested are:

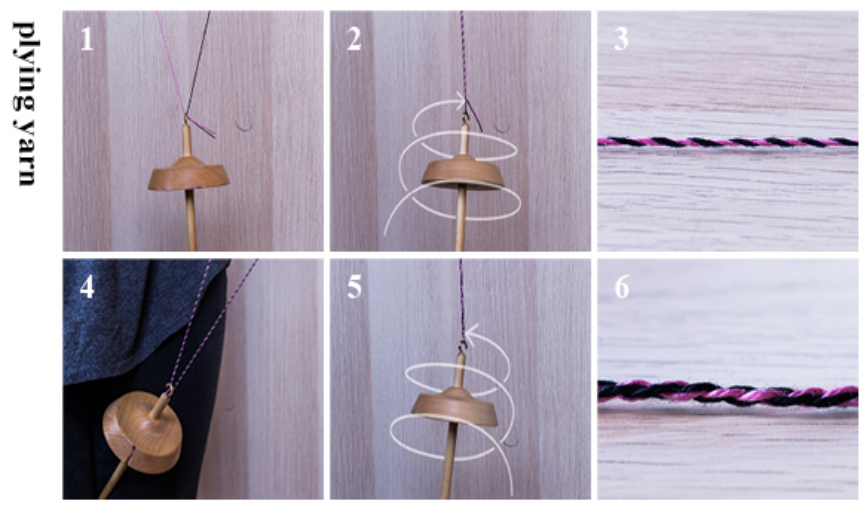

Figure 4: an illustration of the technique we used to ply heating wires with yarns painted with thermochromic pigments.

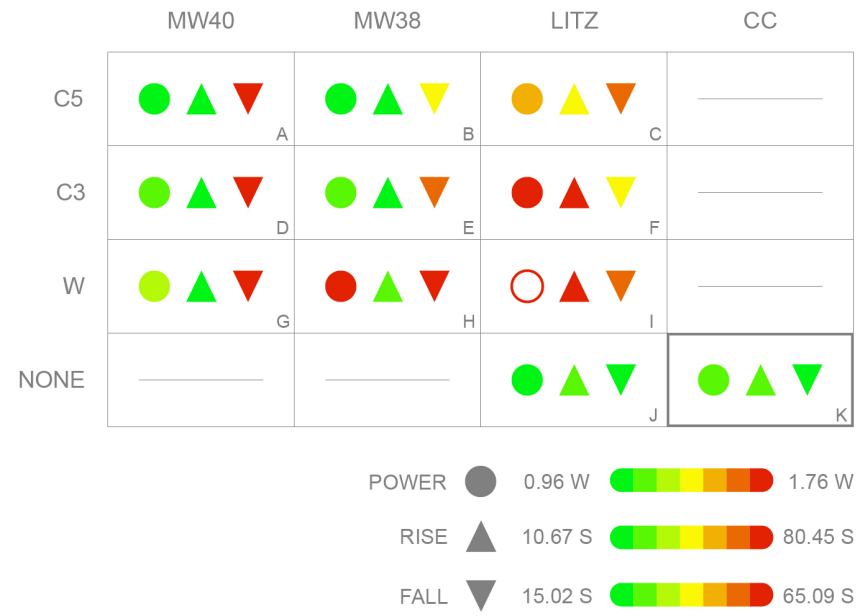

Figure 5: summary of findings from plying various heating wires with traditional findings. The circle represents the power consumption to change from purple to white, relative to the other yarns. The up and down arrows represent relative time for the color change to activate (rise) and deactivate (fall), respectively.

- W: 3/8 Zephyr Wool, color: natural

- C5: 5/2 Pearl Cotton, color: natural

- C3: 3/2 Pearl Cotton, color: natural.

The conductive yarns and wires we tested are:

- MW40: 40 AWG magnet wire

- MW38: 38 AWG magnet wire

- Litz: 36 AWG Litz wire

- CC: Cotton Covered non-insulated Copper

- SS: Plied Stainless Steel

We measured the amount of power required by each yarn to achieve full color change, the quality of the color change, and the time to achieve that change (the rise time) and recover from it (the fall time). When testing the yarns, we pulled them straight, so that they were not looped on themselves, which affects how the yarn heats. In practice, however, yarns that are placed next to each other will heat more quickly than yarns with more air around them, so our measurements represent the upper bounds of power consumption.

Our measurements should be treated as relative, since combinations of handspun fibers and wires, coated with different mixtures and kinds of pigments, tested in different environments and configurations, will vary. Similarly, it can be difficult to tell, exactly, when a yarn has transitioned from one color to the next, which adds some margin of error into our timing measurements. In general, we took measurements when the yarn had completely turned color along its entire length, which in some cases was different than when it started to change. We provide our complete data in auxiliary materials and a summary in Figure 5. Some general tends emerged from our data include: 


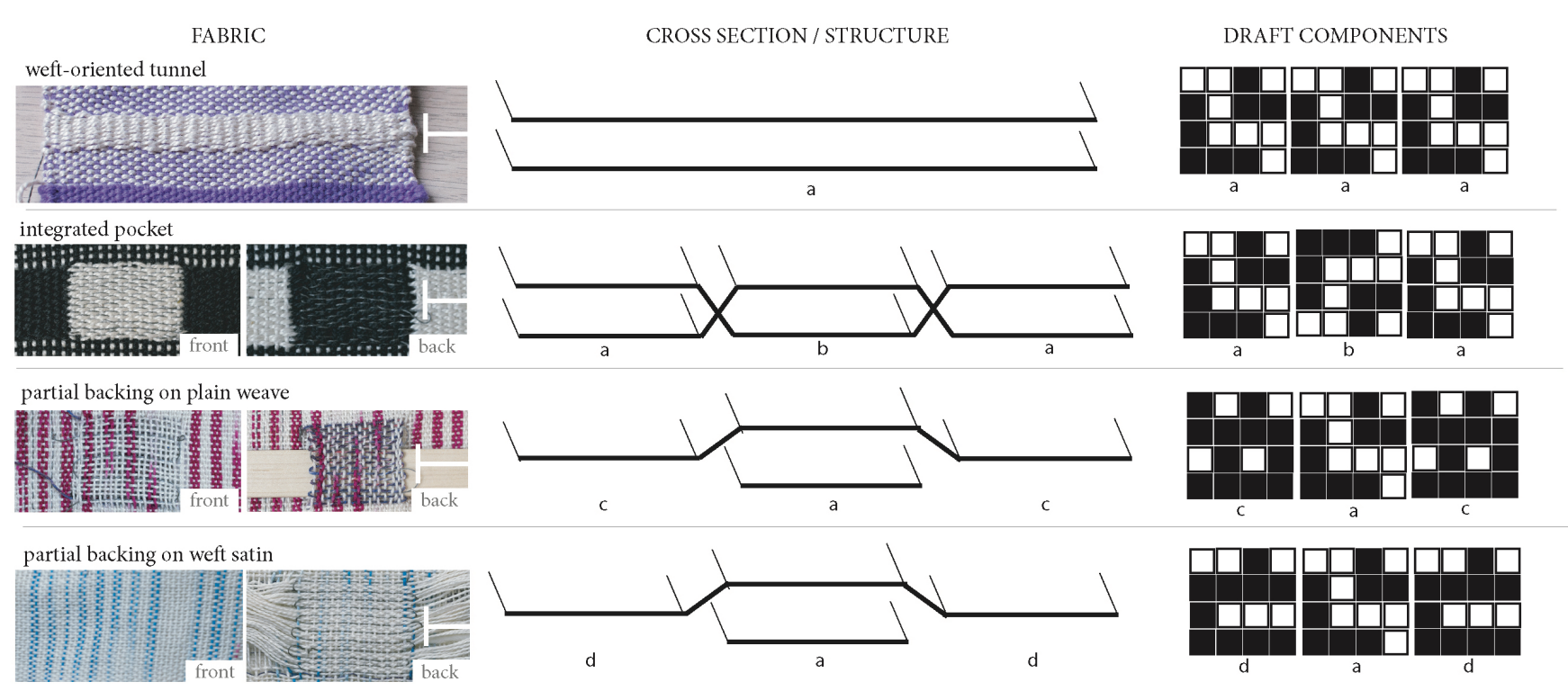

Figure 6: Illustration of double weave structures. The structural view represents the layers of the weave as one would see them if they could slice the fabric and look at the cross-section. The circles mark layer associations of the warp yarns, the red and blue yarns represent how the weft yarns are integrated. The rows are woven in the order they appear in the draft: red, blue, light red, light blue. Repeating those four stitches produces the square sections depicted on the physical sample view.

Many yarns that were plied required less power but had longer rise and fall times. We believe this is because plying created thicker yarns, requiring more heat to travel through the fibers to activate color change. Similarly, there was less air around the fibers to help with cooling.

The cotton yarns changed color more uniformly than wool yarns. Our theory here is that since wool is a crimped fiber, wool yarns contain many pockets of air of various sizes. These air pockets are not uniformly distributed through the yarn and thus, the length of yarn heats unevenly.

Litz had some advantages in terms of quick heating, when it was coated alone rather than spun with other fibers. However, Litz wire is quite expensive and internally contains three very thin enameled copper wires, making it difficult to solder.

The silk insulation on the Litz wires significantly hindered heat transfer when plied with other fibers. The Litz+W combination was our poorest performing because we believe the silk covering the multiple strands of magnet wire absorbed the heat intended for the wool. The timings were so much worse than the others that we threw them out of our measurements, as indicated by the open red circle in the graph (Figure 5i).

The MW40 combinations performed well but were difficult to ply without breaking. The MW38 was the thinnest wire we could ply reliably.

Our best performing yarn, considering all variables, was not hand plied at all, but was CC, a non-insulated copper wire wrapped in white cotton - created by a yarn spinning process performed by a manufacturer. Because the cotton provided all the insulation to the copper, it allows for fast thermal transfer and easy soldering (since one does not have to burn or scratch the enamel off the inner wire prior to soldering). Additionally, the wire itself is very durable while moderately pliable, allowing it to nicely integrate into our fabric without dramatically changing the surface texture.

\section{ADAPTING DOUBLE WEAVING FOR SMART TEXTILES}

Double weaving is a technique that allows a weaver to weave multiple layers at once. It works by splitting the warp yarns into two groups (odd yarns in one group, even yarns in another) and weaving those yarn groupings separately from each other to create two layers that sit atop of each other (Figure 6, top). With this two-layer construction, a weaver can design patterns for each layer separately, allowing for the insertion of conductive yarns into the top and bottom layers in different configurations. For example, [2] uses double weaving to integrate hidden heating elements into a fabric while [23] uses it to embed pressure sensitive pads within the fabric. We experimented with several methods of double weaving during our prototyping and describe them in detail below:

\section{Open or Closed Weft-Oriented Tunnels}

When one switches from a single weave to a double weave as they are weaving, they essentially split a single layer of fabric into two layers. When one switches back to a single layer weave, that double-layered region becomes a tunnel spanning the width of the fabric. The ends of the tunnels can be left open or closed. In the context of smart textiles, this structure makes it easy to route wires through fabrics by running them through the tunnels. 


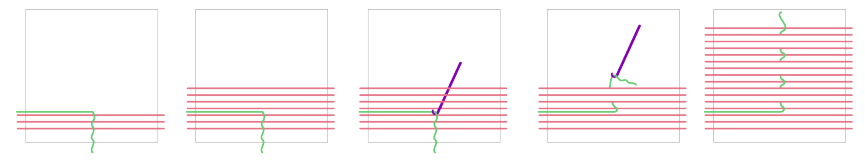

Figure 7: Diagram of our method for partial weft insertion, the insertion of conductive yarn in the warp direction by incrementally pulling the yarn up the fabric with a crochet hook.

\section{Integrated Pockets}

The layer orientations of a double weave can "flip" at arbitrary points, allowing the back face to come to the front face of the fabric and vice versa. Structurally, this "flip" can be used to create pockets that are completely embedded within the fabric. Within such pockets, a designer can place materials or off-the-shelf sensors and completely seal them into the fabric, ensuring that they maintain their position and orientation. We used this technique in early tests to create a pocket with conductive yarn integrated into the top and bottom layers of the pocket and a piece of Velostat, a conductive pressure sensitive material, placed in the middle. However, we found the readings with the Velostat to be too unreliable and considered other methods of creating press buttons. For example, we could have inserted a force-sensor into the pocket to provide more accurate readings, but opted to challenge ourselves with a design that used no existing parts.

\section{Partial Backings}

We refer to the technique we used extensively in our prototype as a partial backing. This double weave only integrates materials to the second layer in the places where they are needed. This structure allowed us to create a seamless and neat front face of the fabric that features the color changing regions while hiding the connective "guts" in the back layer. In early tests, we had integrated partial backings into a fabric that was otherwise plain woven but this created noticeable textural differences in the sensing regions. We updated the design such that the entire top face of the fabric would be woven with the weft satin stitch, (Figures 2 and 6). This made our front facing texture uniform, effectively hiding the sensing regions below it.

The partial backing in our design held rectangular conductive regions for press sensing as well as routing of our conductive wires to the left side of the fabric. We found that two yarns spacing between each conductive yarn was sufficient to ensure our yarns did not inadvertently cross or touch as they were routed through the fabric. A spacing of three yarns between each row of conductive yarn in our touch pad was sufficient for creating reliable press sensing (Figure 8).

\section{Partial Warp Insertion}

To create reliable press buttons, we needed the conductive traces on one layer to routed in the direction of the warp and the other in the weft. Inserting conductive yarns in the warp is more challenging because the warp yarns must be stretched across the entire length of the loom prior to weaving. Similarly, the warp yarns are under much more tension than the weft and we found our conductive stainless yarns to be prone to breaking under this pressure. Since we only needed our conductive traces to travel in the warp direction in small segments, we adapted a technique weavers use to fix broken yarns. In the original technique, one pins the warp yarn to be fixed the fabric in production and then manually pulls it to the back of the loom and attaches a weight to hold it under tension. As one continues weaving rows, the placed warp yarn becomes integrated into the fabric. We realized we could adapt this technique for adding our conductive yarn segments in the warp direction as follows:

We ran one conductive yarn from the left side of the fabric through the bottom layer and pulled it to the top of the fabric when it reached the center point of our button (Figure 7). Then, we continued weaving top and bottom layers as usual. Every three rows, we would use a crochet hook to reach under the top layer of our fabric, and pull the loose thread upward through the length. This allowed us to thread the warp segment along the bottom face of the top layer, ensuring that it could make good contact with the weftoriented conductive pad integrated on the bottom layer while also being invisible to a viewer.

\section{More than Two Layer Weaves}

Our final note on double weaving addresses the number of layers that can be worked at once. While we experimented with and describe the integration of two-layers into a fabric at once, it is possible to weave more than two layers at once. However, for each layer the density of warp yarns is reduced. For instance, in double weaving, the warp yarns are split into two groups (even warps, odd warps) and woven independently of each other. In triple weaving, every $3^{\text {rd }}$ yarn would be used to create a layer, thus increasing the spacing between the warps. We were able to produce a 4layered fabric on our 8-shaft loom. We imagine that these multi-layer structures could lead to more complex integrations of sensing and fabrics, perhaps opening possibilities of multi-layered woven circuitry much like multi-layered programmable circuit boards.

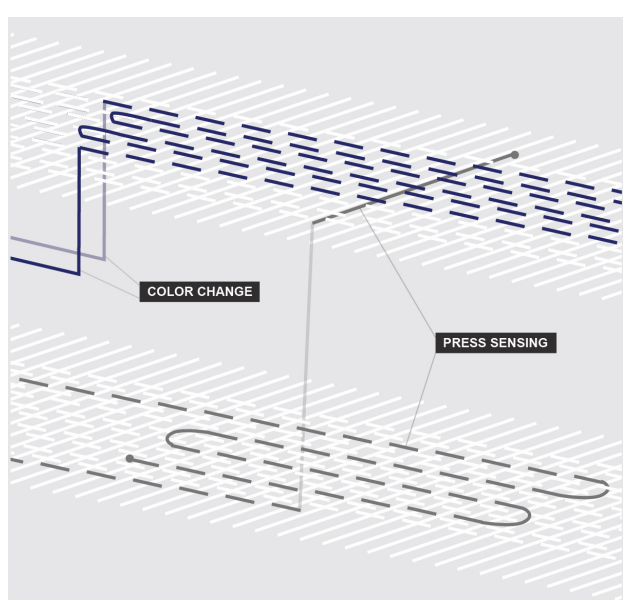

Figure 8: Our fabric leverages double weaving to place color changing regions on the top of the fabric and large conductive pads on the bottom layer. A small piece of conductive yarn is inserted into the top layer to make contact with the bottom pad, completing the circuit. 


\section{FUTURE WORK AND OPEN CHALLENGES}

In prototyping our fabric, we began to develop greater awareness of the struggles that researchers of smart textiles face in terms of sourcing custom yarns as well as understanding the constraints and possibilities of woven structures. We would like to call other researchers to action around some of these challenges, by describing other techniques or possibilities that appear to hold promise for the domain that could be pursued in the future.

\section{Creative Custom Yarns}

Identifying and sourcing the yarns necessary for smart textiles can be difficult and often forces researchers to rely on what they can find rather than what might be ideal for their purpose. In this spirit, we present a few more custom yarn techniques to stimulate the imagination of what might be possible for smart textiles with better control over yarn production:

\section{Rope Making}

A designer can create custom "ropes" by taking several lengths of yarn tied to a peg (or one continuous loom wound around a peg) and tying them to individually rotating hooks. When one turns the hooks with the yarns under tension, all lengths of yarn twisted individually and equally. When one "relaxes" the apparatus, the individual yarn lengths recoil and spin upon each other. The thickness of the resulting rope depends on the thickness of the individual materials that comprise the rope. We tried this technique, using a Schacht Incredible Rope Machine, to combine our yarns and heating wires (Figure 9b). While the resulting yarn had many sections of heating wire per unit length, it was difficult to make ropes without breaking. However, the design of rope making systems can be adapted such that the twisted wires wrap around a "core" that would sit in the center. In this sense, we can envision future research studying how brittle heating (or other) wires could be placed in the core with several lengths of colorchanging threads spun around them.

\section{Knitted Tubes}

We have also conducted several experiments, for a different soft sensor project that used knitted tubes to create a smart string (Figure 9d) [11]. We used a Caron EmbellishKnit hand crank device to knit together a long conductive yarn with a long non-conductive yarn. In this project, we found that smoother or coated conductive yarns worked best to prevent the conductive yarns from grabbing the others. The hollow knitted tube also makes it possible to insert a core. Since the resulting structure is knitted from continuous lengths of yarn, it is quite stretchy and flexible. We also used this structure in the past to create force-sensitive yarns by knitting conductive yarns around a Velostat core, which worked well.

\section{Kumihimo Braiding}

Kumihimo braiding is a technique in which several lengths of yarns are braided into hollow tubes (Figure 9c). Kumihimo templates can be used to create braids with custom textures and patterning. The templates are easy to produce in cardboard on a laser cutter at various

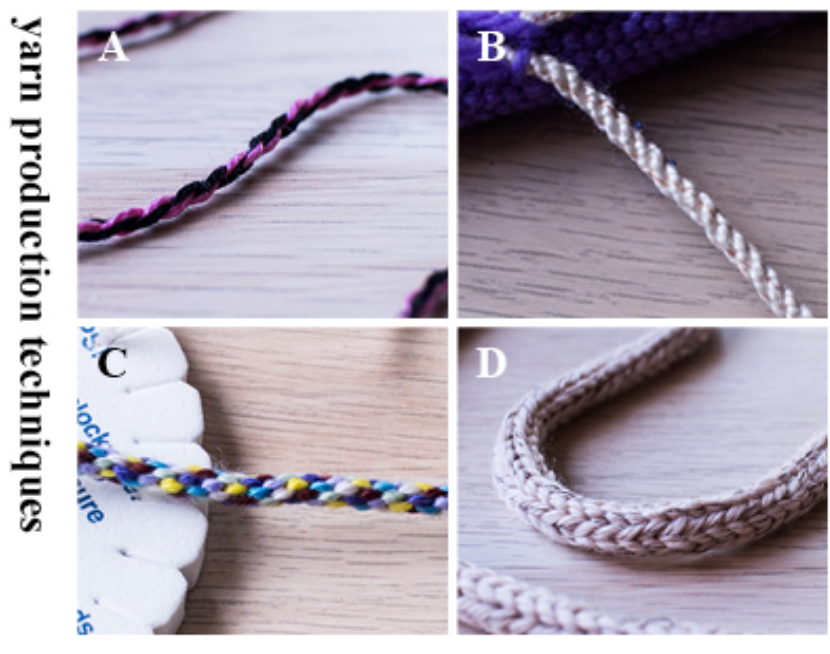

Figure 9: examples of custom yarn structures

thicknesses. During this braiding, the maker has more control over tensions than they might with techniques that spin yarns together, thus, they may be ideal for applications that require brittle materials to be integrated into the outer structure of the yarn.

\section{Possibilities in Double Weaving}

The tunnels and multiple layers afforded by weaving present interesting avenues for future research. For instance, designers could also use these tunnels to integrate off-the-shelf sensors (e.g. long bend sensors) or actuators (e.g. like pneumatic tubes) for easy insertion and removal during testing and prototyping. Tunnels may also be used to insert mechanical tendons or changing materials (shapememory alloys or nylon monofilament-based muscles) that can change the shape of the fabric. If one were to weave several warp and weft facing tunnels, then they could produce a matrix of tunnels through the fabric, again, allowing for the easy insertion and removal of long thin components in two directions instead of one.

What we find most exciting about double weaving is that the pockets can be of arbitrary shape, not just tubes and rectangles, providing that one has a loom with enough sophistication or the hand skills necessary to create complex or non-repeating patterns. For example, existing textiles often use double weaving to alternate the colors that are on the surface of the fabric in given regions. This is why woven fabrics often have the "negative" or "inverse" pattern on the reverse side. What we propose here is that by focusing more on the shape of the tunnels and pockets created through double weaving, apart from the surface patterns they create, we may discover specific uses and possibilities for smart textiles. For example, we envision that pockets can be custom fit to ones specifications and could be used for: routing wires or sensing elements though the innards of a fabric while also creating elegant surface textures; housing components that haven't been realized in woven structures yet, like batteries and microcontrollers; or creating embedded arrays within which hard materials like sensors like magnets, accelerometers or speakers could be inserted into fabrics securely and away from the skin. 


\section{LIMITATIONS}

The prototypes and techniques we demonstrate in this paper certainly have limitations if we were to consider them existing beyond the prototype phase or transitioning into mass-market production. We do not know how the thermochromic yarns we created will do in the wash (we expect badly) largely because we have not yet found an appropriate method for dying the yarns with the pigments we have available (dying chemically bonds the color to the fiber, as opposed to a paint that can wash off). While thermochromic dyes are available, we had trouble finding them in the colors and temperature ranges we desired. Power is also a persistent struggle in textile applications, particularly those that aim to be body worn. While powering every color change region simultaneously would require one to plug into a wall socket, it is feasible to design interactions or optimize power use by limiting the number of sections that receive power at a single time.

\section{REFLECTIONS ON PROTOTYPING BY HAND}

The insights we contribute in this work emerged from a long, slow, humbling, and deeply intriguing process of learning to hand-weave smart textiles. It is a stark contrast from the first author's earlier experience with [5], in which she designed smart textile concepts that a more expert weaver produced, in that it provided a much deeper and embodied knowledge of different weaving structures and possibilities. This knowledge is largely about technique and about the very small and intricate gestures that one makes with their hands when manipulating lengths of string into a fabric. This process of learning what weaving and weavers can do, in both conceptual and embodied senses, has led to a deep admiration and appreciation for those who do it especially well. At the same time, we struggle to understand how to communicate these details, which seem utterly small but critically important, and highlight their importance as research contributions. As the history of smart textiles weaving shows us, the novel ideas for what smart weaving can be have existed for nearly twenty years, what we truly need are ways to effectively communicate the skills necessary in order to develop these ideas.

While some of the techniques we describe here can be reproduced much more quickly and at scale with industrial weaving systems, others like partial warp insertions may be more difficult. While we see how these issues of scale can be cast as limitations, we like to think of them as a specific perspective that makes particular forms of work and personalized innovation possible. Specifically, we are working for a future of smart textiles that are richly crafted or "custom" pieces designed for and with specific users -- a vision that sees smart textiles emerging with ecosystems of custom tailoring and bespoke artifacts that couple personal aesthetics with functionality. Other textiles artists and technologies like Hannah-Perner Wilson and Mika Satomi share this vision, going so far as to opening the first brick and mortar shop "KOBA" for custom smart textiles tailoring services [18]. Bringing these futures into the present requires an embrace of the unique benefits of handcraft and hand-weaving. Technology may not need to

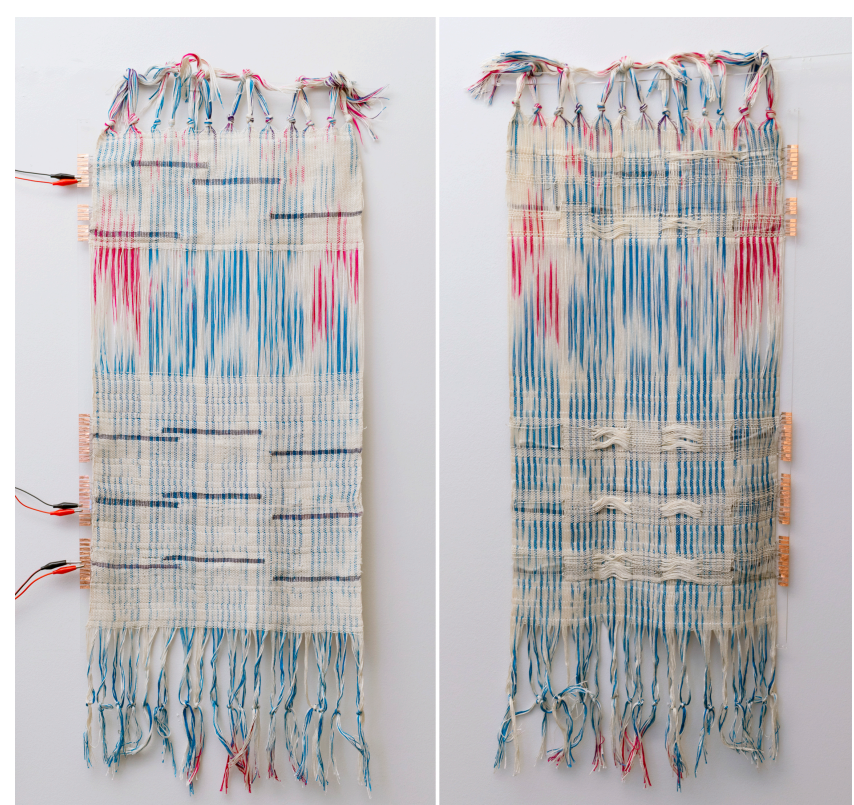

Figure 10: A full picture of the front and back faces of the weave we created with our adapted methods.

be introduced only to make these difficult crafts easier or faster, but could open up new ways of thinking about how we design for more personal or intimate human-technology relationships.

\section{CONCLUSION}

We presented a collection of techniques for double weaving and yarn-playing processes to help developers of smart textiles discover new ways of realizing their smart textile concepts. These techniques emerged through the process of hand-weaving a tapestry that couples several integrated push buttons with pattern changes. In writing this paper, we hope to spur a future in which the technical is more richly crafted.

\section{ACKNOWLEDGMENTS}

A very special thank you to Clement Zheng for assisting with the graphics and diagrams on this paper. Thank you to Shanel Wu, Mikhaila Friske, Andrea Chamorro, Jolie Klefeker, and Christopher Baker for reading and providing feedback. This work is sponsored by NSF \#1755587.

\section{REFERENCES}

[1] Anni Albers. 1974. Anni Albers : On Weaving. Wesleyan, Middletown (Conn.).

[2] Joanna Berzowska. 2004. Very Slowly Animating Textiles: Shimmering Flower. In ACM SIGGRAPH 2004 Sketches (SIGGRAPH '04), 34-. https://doi.org/10.1145/1186223.1186266

[3] Lina M Castano and Alison B Flatau. 2014. Smart fabric sensors and e-textile technologies: a review. Smart Materials and Structures 23, 5: 053001. https://doi.org/10.1088/0964-1726/23/5/053001

[4] Deborah Chandler. 2009. Learning to Weave. Interweave.

[5] Laura Devendorf, Joanne Lo, Noura Howell, Jung Lin Lee, Nan-Wei Gong, M. Emre Karagozler, Shiho 
Fukuhara, Ivan Poupyrev, Eric Paulos, and Kimiko Ryokai. 2016. "I Don't Want to Wear a Screen": Probing Perceptions of and Possibilities for Dynamic Displays on Clothing. In Proceedings of the 2016 CHI Conference on Human Factors in Computing Systems (CHI '16), 6028-6039. https://doi.org/10.1145/2858036.2858192

[6] Allen A. Fannin. 1998. Handloom Weaving Technology: Revised And Updated. Green Editorial, New York.

[7] Noura Howell, Laura Devendorf, Tomás Alfonso Vega Gálvez, Rundong Tian, and Kimiko Ryokai. 2018. Tensions of Data-Driven Reflection: A Case Study of Real-Time Emotional Biosensing. In Proceedings of the 2018 CHI Conference on Human Factors in Computing Systems (CHI '18), 431:1-431:13. https://doi.org/10.1145/3173574.3174005

[8] Ivan Poupyrev, Nan-Wei Gong, Shiho Fukuhara, M. Emre Karagozler, Carsten Schwesig, and Karen Robinson. 2016. Project Jacquard: Manufacturing Digital Textiles at Scale. In Proceedings of the 34th Annual ACM Conference on Human Factors in Computing Systems (CHI '16).

[9] Viirj Kan, Katsuya Fujii, Judith Amores, Chang Long Zhu Jin, Pattie Maes, and Hiroshi Ishii. 2015. Social Textiles: Social Affordances and Icebreaking Interactions Through Wearable Social Messaging. In Proceedings of the Ninth International Conference on Tangible, Embedded, and Embodied Interaction (TEI '15), 619-624. https://doi.org/10.1145/2677199.2688816

[10] Judit Eszter Karpati. Chromosonic. Retrieved from http://chromosonic.tumblr.com/

[11] Josephine Klefeker and Laura Devendorf. String Figuring: A Story of Reflection, Material Inquiry, and a Novel Sensor.

[12] Noura Howell, Laura Devendorf, Rundong Tian, Tomas Vega, Nan-Wei Gong, Ivan Poupyrev, Eric Paulos, and Kimiko Ryokai. 2016. Biosignals as Social Cues: Ambiguity and Emotional Interpretation in
Social Displays of Skin Conductance. In Proceedings of the Designing Interactive Systems Conference.

[13] Maggie Orth. 2009. 100 Electronic Art Years. Retrieved from http://www.maggieorth.com/art_100EAYears.html

[14] Maggie Orth, Rehmi Post, and Ëmily Cooper. 1998. Fabric Computing Interfaces. In CHI 98 Conference Summary on Human Factors in Computing Systems (CHI '98), 331-332. https://doi.org/10.1145/286498.286800

[15] Irene Posch and Ebru Kurbak. 2016. CRAFTED LOGIC Towards Hand-Crafting a Computer. In Proceedings of the 2016 CHI Conference Extended Abstracts on Human Factors in Computing Systems (CHI EA '16), 3881-3884. https://doi.org/10.1145/2851581.2891101

[16] Daniela K. Rosner, Samantha Shorey, Brock R. Craft, and Helen Remick. 2018. Making Core Memory: Design Inquiry into Gendered Legacies of Engineering and Craftwork. In Proceedings of the 2018 CHI Conference on Human Factors in Computing Systems (CHI '18), 531:1-531:13. https://doi.org/10.1145/3173574.3174105

[17] Linda Worbin. 2010. Designing dynamic textile patterns.

[18] KOBA. Retrieved August 4, 2018 from http://www.kobakant.at/KOBA/

[19] Knit Macro-Electronics (2013) | ebru kurbak. Retrieved November 13, 2018 from http://ebrukurbak.net/knitmacro-electronics/

[20] WifiTapestry 1.0. Retrieved August 8, 2018 from http://www.nytimes.com/2015/02/19/arts/international/ when-great-minds-dont-think-alike.html

[21] Woven Signals. aml. Retrieved August 4, 2018 from http://emeteuz.com/woven-signals/

[22] Dynamic Double Weave. Retrieved November 13, 2018 from http://www.maggieorth.com/art_DDW.html

[23] Pressure Sensors : Dena Molnar. Retrieved August 4, 2018 from http://www.denamolnar.com/textiles/pressure-sensors/ 